\title{
On the Mourre estimates for Floquet Hamiltonians
}

$\operatorname{AUTHOR}(S):$

Adachi, Tadayoshi; Kiyose, Amane

CITATION:

Adachi, Tadayoshi ... [et al]. On the Mourre estimates for Floquet

Hamiltonians. Letters in Mathematical Physics 2019, 109(11): 2513-2529

ISSUE DATE:

2019-11

URL:

http://hdl.handle.net/2433/244664

\section{RIGHT:}

This is a post-peer-review, pre-copyedit version of an article published in Letters in Mathematical Physics. The final authenticated version is available online at: http://dx.doi.org/10.1007/s11005-019-01191-x.; The full-text file will be made open to the public on 1 November 2020 in accordance with publisher's 'Terms and Conditions for Self-Archiving'.; This is not the published version. Please cite only the published version.; この論文は出版社版でありません。引用の際

には出版社版をご確認ご利用ください。 


\title{
On the Mourre estimates for Floquet Hamiltonians
}

\author{
Tadayoshi ADACHI
}

Course of Mathematical Science, Department of Human Coexistence

Graduate School of Human and Environmental Studies, Kyoto University

Yoshida-Nihonmatsu-cho, Sakyo-ku, Kyoto-shi, Kyoto 606-8501, Japan

and

Amane KIYOSE

Department of Mathematics, Graduate School of Science, Kobe University

1-1 Rokkodai-cho, Nada-ku, Kobe-shi, Hyogo 657-8501, Japan

Dedicated to Professor Kenji Yajima on the occasion of his 70th birthday

\begin{abstract}
In the spectral and scattering theory for a Schrödinger operator with a time-periodic potential $H(t)=p^{2} / 2+V(t, x)$, the Floquet Hamiltonian $K=-i \partial_{t}+H(t)$ associated with $H(t)$ plays an important role frequently, by virtue of the Howland-Yajima method. In this paper, we introduce a new conjugate operator for $K$ in the standard Mourre theory, that is different from the one due to Yokoyama, in order to relax a certain smoothness condition on $V$.
\end{abstract}

\section{Introduction}

In this paper, we consider the following time-dependent Schrödinger equation

$$
\begin{aligned}
& i \partial_{t} u(t)=H(t) u(t), \quad t \in \boldsymbol{R}, \\
& H(t)=H_{0}+V(t), \quad H_{0}=\frac{1}{2} p^{2} \quad \text { on } \mathscr{H}:=L^{2}\left(\boldsymbol{R}^{d}\right),
\end{aligned}
$$

where $p=-i \nabla_{x}$, and $V(t)$ is the multiplication operator by the real-valued function $V(t, x)$ on $\boldsymbol{R} \times \boldsymbol{R}^{d}$ which is periodic in $t$ with a period $T>0$ :

$$
V(t+T, x)=V(t, x), \quad(t, x) \in \boldsymbol{R} \times \boldsymbol{R}^{d} .
$$


Under some suitable conditions on $V$, the existence and uniqueness of the unitary propagator $U(t, s)$ generated by $H(t)$ can be guaranteed (see e.g. Yajima [23]). In the study of the asymptotic behavior of $U(t, s) \phi, \phi \in \mathscr{H}$, as $t \rightarrow \pm \infty$, we will frequently utilize the so-called Floquet Hamiltonian $K$ associated with $H(t)$ : Let $\boldsymbol{T}=\boldsymbol{R} /(T \boldsymbol{Z})$ be the torus. Set $\mathscr{K}:=L^{2}(\boldsymbol{T} ; \mathscr{H}) \cong L^{2}(\boldsymbol{T}) \otimes L^{2}\left(\boldsymbol{R}^{d}\right)$, and introduce a strongly continuous one-parameter unitary group $\{U(\sigma)\}_{\sigma \in \boldsymbol{R}}$ on $\mathscr{K}$ given by

$$
(\hat{U}(\sigma) \Phi)(t)=U(t, t-\sigma) \Phi(t-\sigma)
$$

for $\Phi \in \mathscr{K}$. By virtue of Stone's theorem, $\hat{U}(\sigma)$ is written as

$$
\hat{U}(\sigma)=e^{-i \sigma K}
$$

with a unique self-adjoint operator $K$ on $\mathscr{K} . K$ is called the Floquet Hamiltonian associated with $H(t)$, and is equal to the natural self-adjoint realization of $-i \partial_{t}+$ $H(t)$. Here we denote by $D_{t}$ the operator $-i \partial_{t}$ with domain $A C(\boldsymbol{T})$, which is the space of absolutely continuous functions on $\boldsymbol{T}$ with their derivatives being square integrable (following the notation in Reed-Simon [17]). As is well-known, $D_{t}$ is self-adjoint on $L^{2}(\boldsymbol{T})$, and its spectrum $\sigma\left(D_{t}\right)$ is equal to $\mathscr{T}:=\omega \boldsymbol{Z}$ with $\omega:=2 \pi / T$. Hence $\boldsymbol{R} \backslash \mathscr{T} \subset \rho\left(D_{t}\right)$ can be decomposed as

$$
\boldsymbol{R} \backslash \mathscr{T}=\bigcup_{n \in \boldsymbol{Z}} I_{n}, \quad I_{n}:=(n \omega,(n+1) \omega)
$$

where $\rho\left(D_{t}\right)=\boldsymbol{C} \backslash \sigma\left(D_{t}\right)$ is the resolvent set of $D_{t}$.

In [24], Yokoyama introduced the self-adjoint operator

$$
\tilde{A}_{1}=\frac{1}{2}\left\{x \cdot p\left(1+p^{2}\right)^{-1}+\left(1+p^{2}\right)^{-1} p \cdot x\right\}
$$

on $\mathscr{K}$ as a conjugate operator for $K$. Roughly speaking, $\tilde{A}_{1}$ is defined by multiplying the generator of dilations

$$
\hat{A}_{0}=\frac{1}{2}(x \cdot p+p \cdot x)
$$

and the resolvent $\left(1+p^{2}\right)^{-1}=\langle p\rangle^{-2}$ of $p^{2}$. He established the following Mourre estimate under some suitable conditions on $V$ : Let $\lambda_{0} \in \boldsymbol{R} \backslash \mathscr{T}$ and $0<\delta<$ $\operatorname{dist}\left(\lambda_{0}, \mathscr{T}\right)$. Put $d_{1}(\lambda):=\operatorname{dist}(\lambda, \mathscr{T} \cap(-\infty, \lambda])$. Then, for any real-valued $f_{\delta} \in C_{0}^{\infty}(\boldsymbol{R})$ supported in $[-\delta, \delta]$, the Mourre estimate

$$
f_{\delta}\left(K-\lambda_{0}\right) i\left[K, \tilde{A}_{1}\right] f_{\delta}\left(K-\lambda_{0}\right) \geq \frac{2\left(d_{1}\left(\lambda_{0}\right)-\delta\right)}{1+2\left(d_{1}\left(\lambda_{0}\right)-\delta\right)} f_{\delta}\left(K-\lambda_{0}\right)^{2}+C_{1, \lambda_{0}, f_{\delta}}
$$


holds with some compact operator $C_{1, \lambda_{0}, f_{\delta}}$ on $\mathscr{K}$. (1.8) which we have given above is slightly better than the estimate obtained in [24]

$f_{\delta}\left(K-\lambda_{0}\right) i\left[K, \tilde{A}_{1}\right] f_{\delta}\left(K-\lambda_{0}\right) \geq \frac{2\left(\operatorname{dist}\left(\lambda_{0}, \mathscr{T}\right)-\delta\right)}{1+2\left(\operatorname{dist}\left(\lambda_{0}, \mathscr{T}\right)-\delta\right)} f_{\delta}\left(K-\lambda_{0}\right)^{2}+C_{1, \lambda_{0}, f_{\delta}}^{\prime}$

with some compact operator $C_{1, \lambda_{0}, f_{\delta}}^{\prime}$ on $\mathscr{K}$, since $\operatorname{dist}\left(\lambda_{0}, \mathscr{T}\right)$ is less than or equal to $d_{1}\left(\lambda_{0}\right)$. Then the standard Mourre theory (see e.g. Cycon-Froese-KirschSimon [6], Amrein-Boutet de Monvel-Georgescu [5] and so on) yields the following spectral properties of $K$ which are important in the scattering theory: The eigenvalues of $K$ in $\boldsymbol{R} \backslash \mathscr{T}$ are of finite multiplicity, and can accumulate only at $\mathscr{T} . \mathscr{T} \cup \sigma_{\mathrm{pp}}(K)$ is a countable closed set. Moreover, the limiting absorption principle for $K$ holds: Let $s>1 / 2$, and $I$ be a compact interval in $\boldsymbol{R} \backslash\left(\mathscr{T} \cup \sigma_{\mathrm{pp}}(K)\right)$. Then, for instance, one has

$$
\sup _{\substack{\operatorname{Re} z \in I \\ \operatorname{Im} z \neq 0}}\left\|\left\langle\tilde{A}_{1}\right\rangle^{-s}(K-z)^{-1}\left\langle\tilde{A}_{1}\right\rangle^{-s}\right\|_{\boldsymbol{B}(\mathscr{K})}<\infty .
$$

Here $\langle x\rangle=\sqrt{1+|x|^{2}}$.

In this paper, we will propose an alternative conjugate operator for $K$ at a nonthreshold energy $\lambda_{0}$ : Let $\lambda_{0} \in \boldsymbol{R} \backslash \mathscr{T}$. Then there exists a unique $n_{\lambda_{0}} \in \boldsymbol{Z}$ such that $\lambda_{0} \in I_{n_{\lambda_{0}}}$. Take $\delta$ as $0<\delta<\operatorname{dist}\left(\lambda_{0}, \mathscr{T}\right)$. Since $\lambda_{0}-\delta \in I_{n_{\lambda_{0}}}$, it is obvious that $\lambda_{0}-\delta \in \boldsymbol{R} \backslash \mathscr{T} \subset \rho\left(D_{t}\right)$. Then, for the sake of obtaining the Mourre estimate for $K$ at $\lambda_{0}$, we introduce the self-adjoint operator

$$
A_{\lambda_{0}, \delta}=\left(\lambda_{0}-\delta-D_{t}\right)^{-1} \otimes \hat{A}_{0}
$$

on $\mathscr{K} \cong L^{2}(\boldsymbol{T}) \otimes L^{2}\left(\boldsymbol{R}^{d}\right)$, by multiplying $\hat{A}_{0}$ and the resolvent $\left(\lambda_{0}-\delta-D_{t}\right)^{-1}$ of $D_{t}$ instead of $\langle p\rangle^{-2}$. Here we note that $\left(\lambda_{0}-\delta-D_{t}\right)^{-1}$ is bounded and self-adjoint. One of the basic properties of $A_{\lambda_{0}, \delta}$ is that

$$
\begin{aligned}
i\left[K_{0}, A_{\lambda_{0}, \delta}\right] & =\left(\lambda_{0}-\delta-D_{t}\right)^{-1} p^{2} \\
& =\left(\lambda_{0}-\delta-D_{t}\right)^{-1}\left\{2\left(K_{0}-D_{t}\right)\right\}, \\
i\left[i\left[K_{0}, A_{\lambda_{0}, \delta}\right], A_{\lambda_{0}, \delta}\right] & =\left(\lambda_{0}-\delta-D_{t}\right)^{-2}\left\{4\left(K_{0}-D_{t}\right)\right\}
\end{aligned}
$$

hold, where $K_{0}=D_{t}+H_{0}$ is the free Floquet Hamiltonian. This yields the fact that

$$
i\left[K_{0}, A_{\lambda_{0}, \delta}\right]\left\langle K_{0}\right\rangle^{-1}, \quad i\left[i\left[K_{0}, A_{\lambda_{0}, \delta}\right], A_{\lambda_{0}, \delta}\right]\left\langle K_{0}\right\rangle^{-1}
$$

are bounded.

Next we impose the following condition $(V)$ on $V$ under consideration:

$(V) V(t, x)$ is a real-valued function on $\boldsymbol{R} \times \boldsymbol{R}^{d}$, is $T$-periodic in $t$, and is decomposed into the sum of $V^{\operatorname{sing}}(t, x)$ and $V^{\text {reg }}(t, x)$, which are also $T$-periodic in 
$t$. If $d<3$, then $V^{\text {sing }}=0$. If $d \geq 3$, then $V^{\operatorname{sing}}(t, \cdot)$ belongs to $C\left(\boldsymbol{R}, L^{q_{0}}\left(\boldsymbol{R}^{d}\right)\right)$ with some $q_{0}>d$, and $\operatorname{supp} V^{\text {sing }}(t, \cdot)$ 's are included in a common compact subset of $\boldsymbol{R}^{d} \cdot\left(\partial_{t} V^{\text {sing }}\right)(t, \cdot)$ and $\left|\left(\nabla V^{\text {sing }}\right)(t, \cdot)\right|$ belong to $C\left(\boldsymbol{R}, L^{q_{1}}\left(\boldsymbol{R}^{d}\right)\right)$ with some $q_{1}>d / 2$, where if $d=3$, then we define $q_{1}$ by $1 / q_{1}=1 /\left(2 q_{0}\right)+1 / 2$. On the other hand, $V^{\text {reg }}(t, x)$ belongs to $C^{2}\left(\boldsymbol{R} \times \boldsymbol{R}^{d}\right)$, and satisfies the decaying conditions

$$
\sup _{t \in \boldsymbol{R}}\left|\left(\partial_{t}^{k} \partial_{x}^{\alpha} V^{\mathrm{reg}}\right)(t, x)\right| \leq C\langle x\rangle^{-\rho-(k+|\alpha|)}, \quad k+|\alpha| \leq 2
$$

with some $\rho>0$.

As for the singular part $V^{\operatorname{sing}}(t, x)$ of $V(t, x)$, we mainly suppose that it has a local singularity like $|x|^{-\gamma}$ with $\gamma>0$, as in e.g. Adachi-Kimura-Shimizu [4]. If $d \geq 3$, then the local singularity like $|x|^{-1+\epsilon}$ with $0<\epsilon<1$ can be permitted by $(V)$. Unfortunately the Coulomb singularity like $|x|^{-1}$ cannot be dealt with in our analysis. Under the condition $(V)$, the existence and uniqueness of the unitary propagator $U(t, s)$ generated by $H(t)$ can be guaranteed by the results of Yajima [23]. It can be also guaranteed that

$$
\left\langle K_{0}\right\rangle^{-1 / 2} i\left[V, A_{\lambda_{0}, \delta}\right]\left\langle K_{0}\right\rangle^{-1}, \quad\left\langle K_{0}\right\rangle^{-1} i\left[i\left[V, A_{\lambda_{0}, \delta}\right], A_{\lambda_{0}, \delta}\right]\left\langle K_{0}\right\rangle^{-1}
$$

are bounded. In fact, as for the regular part $V^{\text {reg }}$ of $V$, it follows from

$$
\begin{aligned}
& \quad i\left[V^{\mathrm{reg}}, A_{\lambda_{0}, \delta}\right] \\
& =-\left(\lambda_{0}-\delta-D_{t}\right)^{-1}\left((x \cdot \nabla) V^{\mathrm{reg}}\right) \\
& \quad-\left(\lambda_{0}-\delta-D_{t}\right)^{-1}\left(\partial_{t} V^{\mathrm{reg}}\right)\left(\lambda_{0}-\delta-D_{t}\right)^{-1} \hat{A}_{0}, \\
& \quad i\left[i\left[V^{\mathrm{reg}}, A_{\lambda_{0}, \delta}\right], A_{\lambda_{0}, \delta}\right] \\
& =\left(\lambda_{0}-\delta-D_{t}\right)^{-2}\left((x \cdot \nabla)^{2} V^{\mathrm{reg}}\right) \\
& \quad+2\left(\lambda_{0}-\delta-D_{t}\right)^{-2}\left(\partial_{t}(x \cdot \nabla) V^{\mathrm{reg}}\right)\left(\lambda_{0}-\delta-D_{t}\right)^{-1} \hat{A}_{0}, \\
& \quad+\left(\lambda_{0}-\delta-D_{t}\right)^{-2}\left(\partial_{t}^{2} V^{\mathrm{reg}}\right)\left(\lambda_{0}-\delta-D_{t}\right)^{-2} \hat{A}_{0}^{2}
\end{aligned}
$$

that

$$
i\left[V^{\mathrm{reg}}, A_{\lambda_{0}, \delta}\right]\left\langle K_{0}\right\rangle^{-1}, \quad i\left[i\left[V^{\mathrm{reg}}, A_{\lambda_{0}, \delta}\right], A_{\lambda_{0}, \delta}\right]\left\langle K_{0}\right\rangle^{-1}
$$

are bounded. Here we used the fact that

$$
\left\langle D_{t}\right\rangle^{-1 / 2}\langle p\rangle\left\langle K_{0}\right\rangle^{-1}, \quad\left\langle D_{t}\right\rangle^{-1}\langle p\rangle^{2}\left\langle K_{0}\right\rangle^{-1}
$$

are bounded, which can be shown in the same way as in the case of Stark Hamiltonians (see e.g. Simon [19]). Moreover, we see that $\left\langle K_{0}\right\rangle^{-1} i\left[V^{\mathrm{reg}}, A_{\lambda_{0}, \delta}\right]\left\langle K_{0}\right\rangle^{-1}$ is compact, by virtue of the local compactness property of $K_{0}$. On the other hand, as for the singular part $V^{\text {sing }}$ of $V$, by using the fact that

$$
\langle p\rangle^{-1}\left((x \cdot \nabla) V^{\text {sing }}(t)\right)\langle p\rangle^{-1}, \quad\langle p\rangle^{-1}\left(\partial_{t} V^{\text {sing }}(t)\right)\langle p\rangle^{-1}
$$


are bounded in $L^{2}\left(\boldsymbol{R}^{d}\right)$, one can show firstly that $\left\langle K_{0}\right\rangle^{-1 / 2} i\left[V^{\text {sing }}, A_{\lambda_{0}, \delta}\right]\left\langle K_{0}\right\rangle^{-1}$ is bounded. Moreover, we see that $\left\langle K_{0}\right\rangle^{-1} i\left[V^{\text {sing }}, A_{\lambda_{0}, \delta}\right]\left\langle K_{0}\right\rangle^{-1}$ is compact. And, by identifying $i\left[i\left[V^{\text {sing }}, A_{\lambda_{0}, \delta}\right], A_{\lambda_{0}, \delta}\right]$ with

$$
i\left\{i\left[V^{\text {sing }}, A_{\lambda_{0}, \delta}\right] A_{\lambda_{0}, \delta}-A_{\lambda_{0}, \delta} i\left[V^{\text {sing }}, A_{\lambda_{0}, \delta}\right]\right\},
$$

one can show that $\left\langle K_{0}\right\rangle^{-1} i\left[i\left[V^{\text {sing }}, A_{\lambda_{0}, \delta}\right], A_{\lambda_{0}, \delta}\right]\left\langle K_{0}\right\rangle^{-1}$ is also bounded. Here we note that in [24], it was assumed that $V^{\text {reg }} \in C^{\infty}\left(\boldsymbol{R} \times \boldsymbol{R}^{d}\right)$, because the pseudodifferential calculus was needed. Our conjugate operator $A_{\lambda_{0}, \delta}$ can relax the smoothness condition on $V^{\text {reg }}$ considerably.

Then some of the main results of this paper are as follows:

Theorem 1.1. Assume $V$ satisfies $(V)$. Let $\lambda_{0} \in \boldsymbol{R} \backslash \mathscr{T}$. Take $\delta$ as $0<\delta<$ $\operatorname{dist}\left(\lambda_{0}, \mathscr{T}\right)$. Define $A_{\lambda_{0}, \delta}$ by (1.10). Then:

(1) For any real-valued $f_{\delta} \in C_{0}^{\infty}(\boldsymbol{R})$ supported in $[-\delta, \delta]$,

$$
f_{\delta}\left(K-\lambda_{0}\right) i\left[K, A_{\lambda_{0}, \delta}\right] f_{\delta}\left(K-\lambda_{0}\right) \geq 2 f_{\delta}\left(K-\lambda_{0}\right)^{2}+C_{\lambda_{0}, f_{\delta}}
$$

holds with some compact operator $C_{\lambda_{0}, f_{\delta}}$ on $\mathscr{K}$. It follows from this that $\sigma_{\mathrm{pp}}(K) \cap$ $\left[\lambda_{0}-\delta / 2, \lambda_{0}+\delta / 2\right]$ is finite, and the eigenvalues of $K$ in $\left[\lambda_{0}-\delta / 2, \lambda_{0}+\delta / 2\right]$ are of finite multiplicity.

(2) In addition, assume $\lambda_{0} \notin \sigma_{\mathrm{pp}}(K)$. Let $0<\varepsilon<2$. Take $\delta>0$ so small that $\left[\lambda_{0}-2 \delta, \lambda_{0}+2 \delta\right] \subset \boldsymbol{R} \backslash\left(\mathscr{T} \cup \sigma_{\mathrm{pp}}(K)\right)$ and

$$
f_{2 \delta}\left(K-\lambda_{0}\right) i\left[K, A_{\lambda_{0}, 2 \delta}\right] f_{2 \delta}\left(K-\lambda_{0}\right) \geq(2-\varepsilon) f_{2 \delta}\left(K-\lambda_{0}\right)^{2}
$$

holds. Suppose $s>1 / 2$. Then

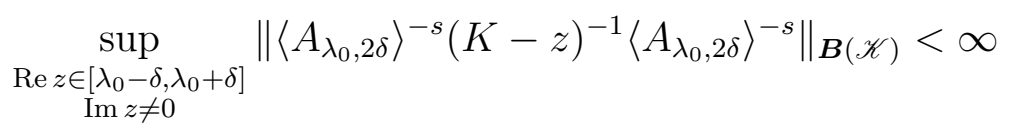

holds. Moreover, $\left\langle A_{\lambda_{0}, 2 \delta}\right\rangle^{-s}(K-z)^{-1}\left\langle A_{\lambda_{0}, 2 \delta}\right\rangle^{-s}$ is a $\boldsymbol{B}(\mathscr{K})$-valued $\theta(s)$-Hölder continuous function on $z \in S_{\lambda_{0}, \delta, \pm}$, where

$$
\begin{aligned}
& \theta(s)=\frac{\min \{s-1 / 2, \rho\}}{\min \{s-1 / 2, \rho\}+1}, \\
& S_{\lambda_{0}, \delta, \pm}=\left\{\zeta \in \boldsymbol{C} \mid \operatorname{Re} \zeta \in\left[\lambda_{0}-\delta, \lambda_{0}+\delta\right], 0< \pm \operatorname{Im} \zeta \leq 1\right\} .
\end{aligned}
$$

And, there exist the norm limits

$$
\left\langle A_{\lambda_{0}, 2 \delta}\right\rangle^{-s}(K-(\lambda \pm i 0))^{-1}\left\langle A_{\lambda_{0}, 2 \delta}\right\rangle^{-s}=\lim _{\epsilon \rightarrow+0}\left\langle A_{\lambda_{0}, 2 \delta}\right\rangle^{-s}(K-(\lambda \pm i \epsilon))^{-1}\left\langle A_{\lambda_{0}, 2 \delta}\right\rangle^{-s}
$$

in $\boldsymbol{B}(\mathscr{K})$ for any $\lambda \in\left[\lambda_{0}-\delta, \lambda_{0}+\delta\right] .\left\langle A_{\lambda_{0}, 2 \delta}\right\rangle^{-s}(K-(\lambda \pm i 0))^{-1}\left\langle A_{\lambda_{0}, 2 \delta}\right\rangle^{-s}$ are also $\theta(s)$-Hölder continuous in $\lambda$. 
Corollary 1.2. Assume $V$ satisfies $(V)$. Then:

(1) The eigenvalues of $K$ in $\boldsymbol{R} \backslash \mathscr{T}$ can accumulate only at $\mathscr{T}$. Moreover, $\mathscr{T} \cup$ $\sigma_{\mathrm{pp}}(K)$ is a countable closed set.

(2) Let I be a compact interval in $\boldsymbol{R} \backslash\left(\mathscr{T} \cup \sigma_{\mathrm{pp}}(K)\right)$. Suppose $1 / 2<s \leq 1$. Then

$$
\sup _{\substack{\operatorname{Re} z \in I \\ \operatorname{Im} z \neq 0}}\left\|\langle x\rangle^{-s}(K-z)^{-1}\langle x\rangle^{-s}\right\|_{\boldsymbol{B}(\mathscr{K})}<\infty
$$

holds. Moreover, $\langle x\rangle^{-s}(K-z)^{-1}\langle x\rangle^{-s}$ is a $\boldsymbol{B}(\mathscr{K})$-valued $\theta(s)$-Hölder continuous function on $z \in S_{I, \pm}$, where

$$
S_{I, \pm}=\{\zeta \in C \mid \operatorname{Re} \zeta \in I, 0< \pm \operatorname{Im} \zeta \leq 1\} .
$$

And, there exist the norm limits

$$
\langle x\rangle^{-s}(K-(\lambda \pm i 0))^{-1}\langle x\rangle^{-s}=\lim _{\epsilon \rightarrow+0}\langle x\rangle^{-s}(K-(\lambda \pm i \epsilon))^{-1}\langle x\rangle^{-s}
$$

in $\boldsymbol{B}(\mathscr{K})$ for $\lambda \in I .\langle x\rangle^{-s}(K-(\lambda \pm i 0))^{-1}\langle x\rangle^{-s}$ are also $\theta(s)$-Hölder continuous in $\lambda$.

In order to obtain Corollary 1.2, we use the argument due to Perry-SigalSimon [16], and the boundedness of

$$
A_{\lambda_{0}, 2 \delta}\left(K-\lambda_{0}-i\right)^{-1}\langle x\rangle^{-1}
$$

which follows from the fact that $\left\langle D_{t}\right\rangle^{-1}\left(K-\lambda_{0}-i\right)^{-1}\langle p\rangle^{2}$ is bounded. By virtue of this, one can show that

$$
A_{\lambda_{0}, 2 \delta}\left(K-\lambda_{0}-i\right)^{-1}\langle p\rangle\langle x\rangle^{-1}, \quad A_{\lambda_{0}, 2 \delta}\left(K-\lambda_{0}-i\right)^{-1}\left\langle D_{t}\right\rangle^{1 / 2}\langle x\rangle^{-1}
$$

are also bounded. Then one may expect that the limiting absorption principle

$$
\sup _{\substack{\operatorname{Re} z \in I \\ \operatorname{Im} z \neq 0}}\left\|\langle x\rangle^{-s} \mathcal{D}^{s}(K-z)^{-1} \mathcal{D}^{s}\langle x\rangle^{-s}\right\|_{\boldsymbol{B}(\mathscr{K})}<\infty
$$

will also hold, where the unbounded 'weight' $\mathcal{D}=\langle p\rangle+\left\langle D_{t}\right\rangle^{1 / 2}$ is equivalent to the 'weight' $\mathscr{D}^{1 / 2}=\left(\langle p\rangle^{4}+\left\langle D_{t}\right\rangle^{2}\right)^{1 / 4}$, which was introduced in KuwabaraYajima [11] for the sake of obtaining a refined limiting absorption principle for $K$. But we have not proved this yet, unfortunately. It is caused by the unboundedness of

$$
\left(K-\lambda_{0}-i\right)^{-1}\langle p\rangle\langle x\rangle^{-1}, \quad\left(K-\lambda_{0}-i\right)^{-1}\left\langle D_{t}\right\rangle^{1 / 2}\langle x\rangle^{-1} .
$$

Instead of the above limiting absorption principle, one can obtain

$$
\sup _{\substack{\operatorname{Re} z \in I \\ \operatorname{Im} z \neq 0}}\left\|\left\langle D_{t}\right\rangle^{-s / 2}\langle x\rangle^{-s}\langle p\rangle^{s}(K-z)^{-1}\langle p\rangle^{s}\langle x\rangle^{-s}\left\langle D_{t}\right\rangle^{-s / 2}\right\|_{\boldsymbol{B}(\mathscr{K})}<\infty
$$


from (1.15) immediately. As for the $N$-body Floquet Hamiltonians, a refined limiting absorption principle for $K$

$$
\sup _{\substack{\operatorname{Re} z \in I \\ \operatorname{Im} z \neq 0}}\left\|\langle x\rangle^{-s}\langle p\rangle^{r}(K-z)^{-1}\langle p\rangle^{r}\langle x\rangle^{-s}\right\|_{\boldsymbol{B}(\mathscr{K})}<\infty
$$

with $0 \leq r<1 / 2<s \leq 1$ was obtained in Møller-Skibsted [14]. They used an extended Mourre theory due to Skibsted [20], and took a 'conjugate operator' for $K$ in the theory as $\hat{A}_{0}$. However, we would like to stick to find a candidate of a conjugate operator for $K$ not in an extended but in the standard Mourre theory, because it seems much easier to obtain some useful propagation estimates for $K$ by applying the standard one.

The plan of this paper is as follows: In $\S 2$, we will give the proof of Theorem 1.1 , in particular, (1.13). In $\S 3$, as an application of our results, we will deal with the problem of the asymptotic completeness for the so-called AC Stark Hamiltonians in the short-range case, although the result was already obtained in [22] and [24]. In $\S 4$, we will make some remarks on the extension to the many body case.

\section{Acknowledgement}

The first author is partially supported by the Grant-in-Aid for Scientific Research (C) \#17K05319 from JSPS. The authors are grateful to the referees for many valuable comments and suggestions.

\section{Proof of Theorem 1.1}

In this section, we prove Theorem 1.1. Here we will give the proof of the Mourre estimate (1.13) only, because the other results can be shown directly by the standard Mourre theory.

As is well-known, $A C(\boldsymbol{T}) \otimes C_{0}^{\infty}\left(\boldsymbol{R}^{d}\right)$ is a core for $K_{0}$, and $D_{t} \otimes \mathrm{Id}+\mathrm{Id} \otimes H_{0}$ defined on $A C(\boldsymbol{T}) \otimes C_{0}^{\infty}\left(\boldsymbol{R}^{d}\right)$ is essentially self-adjoint and its closure is equal to $K_{0}$. If $V$ satisfies the condition $(V)$, then $K$ is self-adjoint with the domain $\mathscr{D}\left(K_{0}\right)$, and $D_{t} \otimes \mathrm{Id}+\mathrm{Id} \otimes H(t)$ defined on $A C(\boldsymbol{T}) \otimes C_{0}^{\infty}\left(\boldsymbol{R}^{d}\right)$ is essentially self-adjoint and its closure is equal to $K$.

Now we will show

$$
\sup _{|\sigma| \leq 1}\left\|K_{0} e^{i \sigma A_{\lambda_{0}, \delta}}\left(K_{0}+i\right)^{-1}\right\|_{\mathscr{B}(\mathscr{K})}<\infty
$$

with $\lambda_{0} \in \boldsymbol{R} \backslash \mathscr{T}$ and $0<\delta<\operatorname{dist}\left(\lambda_{0}, \mathscr{T}\right)$. First of all, we note that the direct integral decomposition of $\left(K_{0}+i\right)^{-1}$ can be given by

$$
\left(K_{0}+i\right)^{-1}=\bigoplus_{k \in \boldsymbol{Z}}\left(k \omega+H_{0}+i\right)^{-1}
$$


and that $e^{i \sigma A_{\lambda_{0}, \delta}}\left(K_{0}+i\right)^{-1} e^{-i \sigma A_{\lambda_{0}, \delta}}$ with $|\sigma| \leq 1$ can be represented as

$$
e^{i \sigma A_{\lambda_{0}, \delta}}\left(K_{0}+i\right)^{-1} e^{-i \sigma A_{\lambda_{0}, \delta}}=\bigoplus_{k \in \boldsymbol{Z}}\left(k \omega+e^{-2 \sigma /\left(\lambda_{0}-\delta-k \omega\right)} H_{0}+i\right)^{-1} .
$$

For the sake of estimating $\left\|\left(k \omega+H_{0}\right)\left(k \omega+e^{-2 \sigma /\left(\lambda_{0}-\delta-k \omega\right)} H_{0}+i\right)^{-1}\right\|_{\mathscr{B}(\mathscr{H})}$, we will introduce the function

$$
\eta_{\sigma}(\kappa, \tau)=\frac{(\tau+\kappa)^{2}}{\left(\tau+e^{-2 \sigma /\left(\lambda_{0}-\delta-\tau\right)} \kappa\right)^{2}+1}
$$

on $[0, \infty) \times \boldsymbol{R}$. Here we note

$$
\left(\partial_{\kappa} \eta_{\sigma}\right)(\kappa, \tau)=\frac{2(\tau+\kappa)\left\{\left(\tau+e^{-2 \sigma /\left(\lambda_{0}-\delta-\tau\right)} \kappa\right)\left(1-e^{-2 \sigma /\left(\lambda_{0}-\delta-\tau\right)}\right) \tau+1\right\}}{\left\{\left(\tau+e^{-2 \sigma /\left(\lambda_{0}-\delta-\tau\right)} \kappa\right)^{2}+1\right\}^{2}} .
$$

Firstly we consider the case where $\tau=k \omega$ with $k \in \boldsymbol{Z} \cap(0, \infty)$. Suppose $1-e^{-2 \sigma /\left(\lambda_{0}-\delta-k \omega\right)} \geq 0$. Since $\left(\partial_{\kappa} \eta_{\sigma}\right)(\kappa, k \omega)>0$ on $[0, \infty)$,

$$
\eta_{\sigma}(\kappa, k \omega) \leq \lim _{\tilde{\kappa} \rightarrow \infty} \eta_{\sigma}(\tilde{\kappa}, k \omega)=e^{4 \sigma /\left(\lambda_{0}-\delta-k \omega\right)} \leq e^{4 /\left(\operatorname{dist}\left(\lambda_{0}, \mathscr{T}\right)-\delta\right)}
$$

holds. Suppose $1-e^{-2 \sigma /\left(\lambda_{0}-\delta-k \omega\right)}<0$. If a zero

$$
\kappa_{0, \sigma}(k \omega)=\frac{e^{2 \sigma /\left(\lambda_{0}-\delta-k \omega\right)}\left\{1-\left(e^{-2 \sigma /\left(\lambda_{0}-\delta-k \omega\right)}-1\right)(k \omega)^{2}\right\}}{\left(e^{-2 \sigma /\left(\lambda_{0}-\delta-k \omega\right)}-1\right) k \omega}
$$

of $\left(\partial_{\kappa} \eta_{\sigma}\right)(\kappa, k \omega)$ belongs to $[0, \infty)$, then

$$
\begin{aligned}
\eta_{\sigma}(\kappa, k \omega) & \leq \eta_{\sigma}\left(\kappa_{0, \sigma}(k \omega), k \omega\right) \\
& =\frac{\left\{e^{2 \sigma /\left(\lambda_{0}-\delta-k \omega\right)}+\left(e^{2 \sigma /\left(\lambda_{0}-\delta-k \omega\right)}+e^{-2 \sigma /\left(\lambda_{0}-\delta-k \omega\right)}-2\right)(k \omega)^{2}\right\}^{2}}{1+\left(e^{-2 \sigma /\left(\lambda_{0}-\delta-k \omega\right)}-1\right)^{2}(k \omega)^{2}} \\
& =e^{4 \sigma /\left(\lambda_{0}-\delta-k \omega\right)}\left\{1+\left(e^{-2 \sigma /\left(\lambda_{0}-\delta-k \omega\right)}-1\right)^{2}(k \omega)^{2}\right\} \\
& =e^{4 \sigma /\left(\lambda_{0}-\delta-k \omega\right)}+4 e^{2 \sigma /\left(\lambda_{0}-\delta-k \omega\right)} \sinh ^{2}\left(\sigma /\left(\lambda_{0}-\delta-k \omega\right)\right)(k \omega)^{2} \\
& \leq e^{4 /\left(\operatorname{dist}\left(\lambda_{0}, \mathscr{T}\right)-\delta\right)}+4 e^{2 /\left(\operatorname{dist}\left(\lambda_{0}, \mathscr{T}\right)-\delta\right)} \sinh ^{2}\left(1 /\left(\lambda_{0}-\delta-k \omega\right)\right)(k \omega)^{2} \\
& \leq e^{4 /\left(\operatorname{dist}\left(\lambda_{0}, \mathscr{T}\right)-\delta\right)}+4 M_{1, \lambda_{0}, \delta} e^{2 /\left(\operatorname{dist}\left(\lambda_{0}, \mathscr{T}\right)-\delta\right)}
\end{aligned}
$$

with

$$
M_{1, \lambda_{0}, \delta}=\sup _{k \in \boldsymbol{Z}}\left\{\sinh ^{2}\left(1 /\left(\lambda_{0}-\delta-k \omega\right)\right)(k \omega)^{2}\right\}<\infty .
$$

Here we used

$$
\lim _{k \rightarrow \pm \infty} \sinh ^{2}\left(1 /\left(\lambda_{0}-\delta-k \omega\right)\right)(k \omega)^{2}=1 .
$$


On the other hand, if $\kappa_{0, \sigma}(k \omega)$ does not belong to $[0, \infty)$, then

$$
\eta_{\sigma}(\kappa, k \omega) \leq \eta_{\sigma}(0, k \omega)=\frac{(k \omega)^{2}}{(k \omega)^{2}+1}<1<e^{4 /\left(\operatorname{dist}\left(\lambda_{0}, \mathscr{T}\right)-\delta\right)}
$$

holds. The case where $\tau=k \omega$ with $k \in Z \cap(-\infty, 0]$ can be also dealt with quite similarly. Finally we have

$$
\eta_{\sigma}(\kappa, k \omega) \leq e^{4 /\left(\operatorname{dist}\left(\lambda_{0}, \mathscr{T}\right)-\delta\right)}+4 M_{1, \lambda_{0}, \delta} e^{2 /\left(\operatorname{dist}\left(\lambda_{0}, \mathscr{T}\right)-\delta\right)}=: M_{2, \lambda_{0}, \delta}^{2}, \quad \kappa \in[0, \infty),
$$

for any $k \in \boldsymbol{Z}$, which yields

$$
\sup _{k \in \boldsymbol{Z}}\left\|\left(k \omega+H_{0}\right)\left(k \omega+e^{-2 \sigma /\left(\lambda_{0}-\delta-k \omega\right)} H_{0}+i\right)^{-1}\right\|_{\mathscr{B}(\mathscr{H})} \leq M_{2, \lambda_{0}, \delta} .
$$

This implies (2.1) because of

$$
\begin{aligned}
& \sup _{|\sigma| \leq 1}\left\|K_{0} e^{i \sigma A_{\lambda_{0}, \delta}}\left(K_{0}+i\right)^{-1}\right\|_{\mathscr{B}(\mathscr{K})} \\
= & \sup _{|\sigma| \leq 1}\left\|K_{0} e^{i \sigma A_{\lambda_{0}, \delta}}\left(K_{0}+i\right)^{-1} e^{-i \sigma A_{\lambda_{0}, \delta}}\right\|_{\mathscr{B}(\mathscr{K})} \leq M_{2, \lambda_{0}, \delta} .
\end{aligned}
$$

Thus we also have

$$
\sup _{|\sigma| \leq 1}\left\|K e^{i \sigma A_{\lambda_{0}, \delta}}(K+i)^{-1}\right\|_{\mathscr{B}(\mathscr{K})}<\infty .
$$

By including the relations between $K$ and $A_{\lambda_{0}, \delta}$ mentioned in $\S 1$, eventually we have completed checking the required conditions on $A_{\lambda_{0}, \delta}$ as a conjugate operator for $K$ in the standard Mourre theory.

Now we will show Theorem 1.1, in particular, the Mourre estimate (1.13). Take a unique $n_{\lambda_{0}} \in \boldsymbol{Z}$ such that $\lambda_{0} \in I_{n_{\lambda_{0}}}$. Let $f_{\delta} \in C_{0}^{\infty}(\boldsymbol{R})$ be real-valued, and be supported in $[-\delta, \delta]$. Under the condition $(V), f_{\delta}\left(K-\lambda_{0}\right)-f_{\delta}\left(K_{0}-\lambda_{0}\right)$ is compact. Since $i\left[K_{0}, A_{\lambda_{0}, \delta}\right]\left\langle K_{0}\right\rangle^{-1}$ is bounded, and $\left\langle K_{0}\right\rangle^{-1} i\left[V, A_{\lambda_{0}, \delta}\right]\left\langle K_{0}\right\rangle^{-1}$ is compact as mentioned in $\S 1$, we have

$$
\begin{aligned}
& f_{\delta}\left(K-\lambda_{0}\right) i\left[K, A_{\lambda_{0}, \delta}\right] f_{\delta}\left(K-\lambda_{0}\right) \\
= & f_{\delta}\left(K-\lambda_{0}\right) i\left[K_{0}, A_{\lambda_{0}, \delta}\right] f_{\delta}\left(K-\lambda_{0}\right) \\
& \quad+f_{\delta}\left(K-\lambda_{0}\right) i\left[V, A_{\lambda_{0}, \delta}\right] f_{\delta}\left(K-\lambda_{0}\right) \\
= & f_{\delta}\left(K_{0}-\lambda_{0}\right) i\left[K_{0}, A_{\lambda_{0}, \delta}\right] f_{\delta}\left(K_{0}-\lambda_{0}\right)+C_{\lambda_{0}, f_{\delta}}^{\prime}
\end{aligned}
$$

with some compact operator $C_{\lambda_{0}, f_{\delta}}^{\prime}$ on $\mathscr{K} \cdot f_{\delta}\left(K_{0}-\lambda_{0}\right) i\left[K_{0}, A_{\lambda_{0}, \delta}\right] f_{\delta}\left(K_{0}-\lambda_{0}\right)$ can be decomposed into the direct integral

$$
\bigoplus_{k \in \boldsymbol{Z}} \frac{2}{\lambda_{0}-\delta-k \omega} H_{0} f_{\delta}\left(H_{0}-\left(\lambda_{0}-k \omega\right)\right)^{2}
$$


Suppose $\lambda_{0}-k \omega<0$, that is, $k \geq n_{\lambda_{0}}+1$. Then $f_{\delta}\left(H_{0}-\left(\lambda_{0}-k \omega\right)\right)=0$ holds because of $H_{0}=p^{2} / 2$. Suppose $\lambda_{0}-k \omega>0$, that is, $k \leq n_{\lambda_{0}}$. Then one can obtain

$$
H_{0} f_{\delta}\left(H_{0}-\left(\lambda_{0}-k \omega\right)\right)^{2} \geq\left(\lambda_{0}-k \omega-\delta\right) f_{\delta}\left(H_{0}-\left(\lambda_{0}-k \omega\right)\right)^{2}
$$

easily. Thus we have

$$
\begin{aligned}
& f_{\delta}\left(K_{0}-\lambda_{0}\right) i\left[K_{0}, A_{\lambda_{0}, \delta}\right] f_{\delta}\left(K_{0}-\lambda_{0}\right) \\
= & \bigoplus_{k \leq n_{\lambda_{0}}} \frac{2}{\lambda_{0}-\delta-k \omega} H_{0} f_{\delta}\left(H_{0}-\left(\lambda_{0}-k \omega\right)\right)^{2} \\
\geq & \bigoplus_{k \leq n_{\lambda_{0}}} 2 f_{\delta}\left(H_{0}-\left(\lambda_{0}-k \omega\right)\right)^{2}=2 f_{\delta}\left(K_{0}-\lambda_{0}\right)^{2}
\end{aligned}
$$

By combining this and (2.6), and using that $f_{\delta}\left(K-\lambda_{0}\right)-f_{\delta}\left(K_{0}-\lambda_{0}\right)$ is compact again, we obtain the Mourre estimate (1.13)

$$
f_{\delta}\left(K-\lambda_{0}\right) i\left[K, A_{\lambda_{0}, \delta}\right] f_{\delta}\left(K-\lambda_{0}\right) \geq 2 f_{\delta}\left(K-\lambda_{0}\right)^{2}+C_{\lambda_{0}, f_{\delta}}
$$

with some compact operator $C_{\lambda_{0}, f_{\delta}}$ on $\mathscr{K}$.

\section{Application}

As an application of our results, we consider a scattering problem for the so-called AC Stark Hamiltonians.

We consider a system of one particle moving in a given time-periodic electric field $E(t) \in \boldsymbol{R}^{d}$. Suppose that $E(t)$ belongs to $C^{0}\left(\boldsymbol{R} ; \boldsymbol{R}^{d}\right)$, and $T$-periodic, that is, $E(t+T)=E(t)$ for any $t \in \boldsymbol{R}$. Moreover, the mean $E_{\mathrm{m}}$ of $E(t)$ in time is zero, that is,

$$
E_{\mathrm{m}}:=\frac{1}{T} \int_{0}^{T} E(t) d t=0 .
$$

A typical example of such $E(t)$ 's is $E_{0} \cos (\omega t)$ with non-zero $E_{0} \in \boldsymbol{R}^{d}$ and $\omega=$ $2 \pi / T$, which was considered in Kitada-Yajima [9]. As for the case where $E_{\mathrm{m}} \neq 0$, see Møller [13] and Adachi-Kimura-Shimizu [4]. Then the Hamiltonian $\hat{H}(t)$ for the system is given by

$$
\hat{H}(t)=\hat{H}_{0}(t)+V(x), \quad \hat{H}_{0}(t)=\frac{1}{2} p^{2}-E(t) \cdot x
$$

on $L^{2}\left(\boldsymbol{R}^{d}\right) . \hat{H}_{0}(t)$ is called the free AC Stark Hamiltonian, and $\hat{H}(t)$ is called an AC Stark Hamiltonian. We denote by $\hat{U}_{0}(t, s)$ and $\hat{U}(t, s)$ the unitary propagators 
generated by $\hat{H}_{0}(t)$ and $\hat{H}(t)$, respectively. Now, as in [13], we define $\boldsymbol{R}^{d}$-valued $T$-periodic functions $b_{0}(t), b(t)$ and $c(t)$ on $\boldsymbol{R}$ by

$$
\begin{aligned}
b_{0}(t):=\int_{0}^{t} E(s) d s, \quad b_{0, \mathrm{~m}}:=\frac{1}{T} \int_{0}^{T} b_{0}(s) d s, \\
b(t):=b_{0}(t)-b_{0, \mathrm{~m}}, \quad c(t):=\int_{0}^{t} b(s) d s .
\end{aligned}
$$

$b_{0}(t)$ is an auxiliary one for the sake of making $c(t) T$-periodic. Here we introduce the time-dependent Hamiltonian

$$
H(t)=H_{0}+V(x+c(t)), \quad H_{0}=\frac{1}{2} p^{2}
$$

on $\mathscr{H}=L^{2}\left(\boldsymbol{R}^{d}\right)$. We denote by $U(t, s)$ the unitary propagator generated by $H(t)$. As is well-known, the following Avron-Herbst formula holds:

$$
\hat{U}_{0}(t, s)=\mathscr{T}(t) e^{-i(t-s) H_{0}} \mathscr{T}(s)^{*}, \quad \hat{U}(t, s)=\mathscr{T}(t) U(t, s) \mathscr{T}(s)^{*}
$$

with

$$
\mathscr{T}(t)=e^{-i a(t)} e^{i b(t) \cdot x} e^{-i c(t) \cdot p}, \quad a(t)=\int_{0}^{t} \frac{1}{2}|b(s)|^{2} d s .
$$

This formula with $E(t)=E_{0} \cos (\omega t)$ was first proved in [9]. Now we will consider the problem of the asymptotic completeness of the wave operators

$$
\hat{W}^{ \pm}=\underset{t \rightarrow \infty}{\operatorname{sim}} \hat{U}(t, 0)^{*} \hat{U}_{0}(t, 0)
$$

for short-range $V$. The asymptotic completeness of $\hat{W}^{ \pm}$is formulated as

$$
\operatorname{Ran}\left(\hat{W}^{ \pm}\right)=L_{\mathrm{c}}^{2}(\hat{U}(T, 0)),
$$

where $L_{\mathrm{c}}^{2}(\hat{U}(T, 0))$ is the continuous spectral subspace of the Floquet operator $\hat{U}(T, 0)$. We impose the following short-range condition $(V)_{\mathrm{SR}}$ on $V$ :

$(V)_{\mathrm{SR}} V(x)$ is a real-valued function on $\boldsymbol{R}^{d}$, and is decomposed into the sum of $\hat{V}^{\text {sing }}(x)$ and $\hat{V}^{\mathrm{SR}}(x)$. If $d<3$, then $\hat{V}^{\text {sing }}=0$. If $d \geq 3$, then $\hat{V}^{\text {sing }}$ belongs to $L^{q_{0}}\left(\boldsymbol{R}^{d}\right)$ with some $q_{0}>d$, and is compactly supported. $\left|\left(\nabla \hat{V}^{\text {sing }}\right)\right|$ belongs to $L^{q_{1}}\left(\boldsymbol{R}^{d}\right)$ with some $q_{1}>d / 2$, where if $d=3$, then we define $q_{1}$ by $1 / q_{1}=$ $1 /\left(2 q_{0}\right)+1 / 2$. $\hat{V}^{\mathrm{SR}}(x)$ belongs to $C^{2}\left(\boldsymbol{R}^{d}\right)$, and satisfies the decaying conditions

$$
\left|\left(\partial_{x}^{\alpha} \hat{V}^{\mathrm{SR}}\right)(x)\right| \leq C\langle x\rangle^{-\rho_{\mathrm{SR}}-|\alpha|}, \quad|\alpha| \leq 2
$$

with some $\rho_{\mathrm{SR}}>1$. 
Here we note that the singular part $\hat{V}^{\text {sing }}$ of $V$ satisfies the same condition posed in [4], but the short-range part $\hat{V}^{\mathrm{SR}}$ of $V$ has to satisfy the condition which is stronger than the one posed in [13] and [4]. It is caused by that the mean of $E(t)$ in time is not non-zero but zero. Under the condition $(V)_{\mathrm{SR}}, V(x+c(t))=$ $\hat{V}^{\text {sing }}(x+c(t))+\hat{V}^{\mathrm{SR}}(x+c(t))$ satisfies the condition $(V)$ with $\rho=\rho_{\mathrm{SR}}-1>0$. Here we note

$$
\begin{aligned}
& \partial_{t}(V(x+c(t)))=b(t) \cdot(\nabla V)(x+c(t)), \\
& \partial_{t}^{2}\left(\hat{V}^{\mathrm{SR}}(x+c(t))\right)=E(t) \cdot\left(\nabla \hat{V}^{\mathrm{SR}}\right)(x+c(t))+b(t)\left(\nabla^{2} \hat{V}^{\mathrm{SR}}\right)(x+c(t)) b(t)^{\mathrm{T}},
\end{aligned}
$$

where $b(t)^{\mathrm{T}}$ stands for the transpose of $b(t)$. Now we also introduce the wave operators

$$
W^{ \pm}=\underset{t \rightarrow \infty}{\operatorname{sim}} U(t, 0)^{*} e^{-i t H_{0}} .
$$

Then it is obvious that the relation between $\hat{W}^{ \pm}$and $W^{ \pm}$

$$
\hat{W}^{ \pm}=\mathscr{T}(0) W^{ \pm} \mathscr{T}(0)^{*}
$$

holds. We note $\mathscr{T}(0)=e^{-i b_{0, \mathrm{~m}} \cdot x}$. Thus the problem of the asymptotic completeness of $\hat{W}^{ \pm}$can be reduced to that of $W^{ \pm}$

$$
\operatorname{Ran}\left(W^{ \pm}\right)=\mathscr{H}_{\mathrm{c}}(U(T, 0)),
$$

where $\mathscr{H}_{\mathrm{c}}(U(T, 0))$ is the continuous spectral subspace of the Floquet operator $U(T, 0)$. Here we used

$$
L_{\mathrm{c}}^{2}(\hat{U}(T, 0))=\mathscr{T}(0) \mathscr{H}_{\mathrm{c}}(U(T, 0)),
$$

because $\mathscr{T}(0) \mathscr{T}(T)^{*}=e^{i a(T)}$ is a scalar.

As is well-known, in the proof of the asymptotic completeness of $W^{ \pm}$, the so-called Howland-Yajima method plays an important role: Introduce the Floquet Hamiltonians $K_{0}$ and $K$ associated with $H_{0}$ and $H(t)$, respectively, and the wave operators

$$
\mathscr{W}^{ \pm}\left(K, K_{0}\right)=\underset{\sigma \rightarrow \pm \infty}{\mathrm{s}-\lim _{\sigma}} e^{i \sigma K} e^{-i \sigma K_{0}} .
$$

After the existence of $W^{ \pm}$has been guaranteed, the asymptotic completeness of $\mathscr{W}^{ \pm}\left(K, K_{0}\right)$ yields that of $W^{ \pm}$. This is the essence of the Howland-Yajima method.

If $\hat{V}^{\text {sing }}=0$, then we have only to use the limiting absorption principle (1.16) in order to show the asymptotic completeness of $\mathscr{W}^{ \pm}\left(K, K_{0}\right)$. In fact, (1.16) yields the local $K$-smoothness of $\langle x\rangle^{-s}$ with $s>1 / 2$ :

$$
\int_{-\infty}^{\infty}\left\|\langle x\rangle^{-s} e^{-i \sigma K} f_{2 \delta}\left(K-\lambda_{0}\right) \Phi\right\|_{\mathscr{K}}^{2} d \sigma \leq C\|\Phi\|_{\mathscr{K}}^{2}
$$


Since the local $K_{0}$-smoothness of $\langle x\rangle^{-s}$ with $s>1 / 2$ can be also obtained, these propagation estimates yield the existence of $\mathscr{W}^{ \pm}\left(K, K_{0}\right)$ and the adjoint wave operators

$$
\mathscr{W}^{ \pm}\left(K_{0}, K\right)=\underset{\sigma \rightarrow \pm \infty}{\mathrm{s}-\lim } e^{i \sigma K_{0}} e^{-i \sigma K} P_{\mathrm{c}}(K)
$$

immediately, where $P_{\mathrm{c}}(K)$ is the spectral projection onto the continuous spectral subspace $\mathscr{K}_{\mathrm{c}}(K)$ of $K$. Thus one can obtain the asymptotic completeness of $\mathscr{W}^{ \pm}$, as is well-known. If $\hat{V}^{\text {sing }} \neq 0$, then we have to avoid the matter caused by its singularity in the proof of the existence of both $\mathscr{W}^{ \pm}\left(K, K_{0}\right)$ and $\mathscr{W}^{ \pm}\left(K_{0}, K\right)$. To this end, we will use the so-called minimal velocity estimate like

$$
\int_{1}^{\infty}\left\|F\left(\frac{|x|}{\sigma} \leq \sqrt{c_{0}\left(d_{2}\left(\lambda_{0}\right)-2 \delta\right)}\right) e^{-i \sigma K} f_{2 \delta}\left(K-\lambda_{0}\right) \Phi\right\|_{\mathscr{K}}^{2} \frac{d \sigma}{\sigma} \leq C\|\Phi\|_{\mathscr{K}}^{2}
$$

with some $c_{0}>0$ and sufficiently small $\varepsilon>0$, which follows from

$$
\int_{1}^{\infty}\left\|F\left(2-4 \varepsilon \leq \frac{A_{\lambda_{0}, 2 \delta}}{\sigma} \leq 2-2 \varepsilon\right) e^{-i \sigma K} f_{2 \delta}\left(K-\lambda_{0}\right) \Phi\right\|_{\mathscr{K}}^{2} \frac{d \sigma}{\sigma} \leq C\|\Phi\|_{\mathscr{K}}^{2} .
$$

These propagation estimates can be proved in the same way as in Sigal-Soffer [18], by virtue of the Mourre estimate (1.14). Here $F(x \in \Omega)$ denotes the characteristic function of the set of $\Omega$, and

$$
d_{2}(\lambda)=\operatorname{dist}\left(\lambda, \mathscr{T} \cup \sigma_{\mathrm{pp}}(K)\right) .
$$

If $d_{2}\left(\lambda_{0}\right)$ in (3.10) could be replaced by

$$
\operatorname{dist}\left(\lambda_{0},\left(\mathscr{T} \cup \sigma_{\mathrm{pp}}(K)\right) \cap\left(-\infty, \lambda_{0}\right]\right),
$$

then (3.10) would become more natural and refined.

In the long-range case, it seems necessary to obtain some refined propagation estimates for $\hat{U}(t, s)$ or $U(t, s)$. Unfortunately, we have not done it yet. The result on the asymptotic completeness was already obtained in Kitada-Yajima [9] via the Enss method. As for the case where $E_{\mathrm{m}} \neq 0$, see Adachi [2] and Adachi-KimuraShimizu [4].

\section{Concluding remarks}

Although we consider the one body case only in this paper, here we will make some remarks on the many body case. 
We consider a system of $N$ particles moving in a given $T$-periodic electric field in $\boldsymbol{R}^{d}$. In the center-of-mass frame, the total Hamiltonian $\hat{H}(t)$ is given as

$$
\hat{H}(t)=-\frac{1}{2} \Delta_{X}-\langle E(t), x\rangle+V, \quad V=\sum_{1 \leq j<k \leq N} V_{j k}\left(x_{j}-x_{k}\right)
$$

on $L^{2}(X)$, where $X$ is the configuration space for the system under consideration in the center-of-mass frame with a certain suitable metric $\langle\cdot, \cdot\rangle, x \in X, \Delta_{X}$ is the Laplace-Beltrami operator on $X, E(t) \in C^{0}(\boldsymbol{R} ; X)$ is $T$-periodic, and $V_{j k}$ 's are pair interactions. If $N=2$, then $\hat{H}(t)$ is essentially the same as that in $\S 3$. Hence we suppose $N \geq 3$. We denote by $\hat{U}(t, s)$ the propagator generated by $\hat{H}(t)$, and put

$$
E_{\mathrm{m}}:=\frac{1}{T} \int_{0}^{T} E(s) d s \in X .
$$

As in Møller [13] and Adachi [1], we define $X$-valued $T$-periodic functions $b_{0}(t)$, $b(t)$ and $c(t)$ on $\boldsymbol{R}$ by

$$
\begin{aligned}
& b_{0}(t):=\int_{0}^{t}\left(E(s)-E_{\mathrm{m}}\right) d s, \quad b_{0, \mathrm{~m}}:=\frac{1}{T} \int_{0}^{T} b_{0}(s) d s, \\
& b(t):=b_{0}(t)-b_{0, \mathrm{~m}}, \quad c(t):=\int_{0}^{t} b(s) d s,
\end{aligned}
$$

and introduce the time-dependent Hamiltonian

$$
H(t)=H_{0}+V(x+c(t)), \quad H_{0}=-\frac{1}{2} \Delta_{X}-\left\langle E_{\mathrm{m}}, x\right\rangle
$$

on $L^{2}(X)$. If $E_{\mathrm{m}} \neq 0$, then $H_{0}$ is called the free $N$-body Stark Hamiltonian. We denote by $U(t, s)$ the unitary propagator generated by $H(t)$. As is well-known, the following Avron-Herbst formula holds:

$$
\hat{U}_{0}(t, s)=\mathscr{T}(t) e^{-i(t-s) H_{0}} \mathscr{T}(s)^{*}, \quad \hat{U}(t, s)=\mathscr{T}(t) U(t, s) \mathscr{T}(s)^{*}
$$

with

$$
\mathscr{T}(t)=e^{-i a(t)} e^{i b(t) \cdot x} e^{-i c(t) \cdot p}, \quad a(t)=\int_{0}^{t}\left(\frac{1}{2}|b(s)|^{2}-\left\langle E_{\mathrm{m}}, c(s)\right\rangle\right) d s,
$$

where $|b(s)|^{2}=\langle b(s), b(s)\rangle$.

When $E_{\mathrm{m}} \neq 0$, in [1] and [2], Adachi already obtained the result of the asymptotic completeness for the system under consideration, both in the short-range and 
the long-range cases, by introducing the Floquet Hamiltonian $K$ associated with $\hat{H}(t)$. As for this $K$,

$$
A=\left\langle\frac{E_{\mathrm{m}}}{\left|E_{\mathrm{m}}\right|},-i \nabla_{X}\right\rangle
$$

is a conjugate operator for $K$ in the standard Mourre theory, where $-i \nabla_{X}$ is the velocity operator on $X$. Here we emphasize that in the case where $N=2$, in [13], Møller proposed this operator as a conjugate operator for $K$ before [1]. Roughly speaking, the conjugate operator due to Møller possesses its natural extension to $N$-body systems. On the other hand, when $E_{\mathrm{m}}=0$, any candidates of a conjugate operator for $K$ in the standard Mourre theory have not been found yet, except in the case where $N=2$. As mentioned above, in the case where $N=2$, Yokoyama proposed a conjugate operator $\tilde{A}_{1}$ for $K$ in [24]. Unfortunately, $\tilde{A}_{1}$ seems not have any natural extension to $N$-body systems. It is caused by the 'factor' $\left(1+p^{2}\right)^{-1}$ of $\tilde{A}_{1}$ (see [14] for the detail). Hence, in [14], Møller and Skibsted took $\hat{A}_{0}$ as a conjugate operator for $K$ in an extended Mourre theory, as mentioned in $\S 1$. As for the study of the asymptotic completeness for three-body AC Stark Hamiltonians via the Faddeev method, see Korotyaev [10] and Nakamura [15].

Our aim of this paper is to replace the factor $\left(1+p^{2}\right)^{-1}$ by some other appropriate one in order to let a conjugate operator possess its extension to $N$-body systems. However, we have not accomplished this aim yet, unfortunately. We have to deal with the term like

$$
-\left(\lambda_{0}-\delta-D_{t}\right)^{-1}\left\langle b(t),\left(\nabla_{X} V\right)(x+c(t))\right\rangle\left(\lambda_{0}-\delta-D_{t}\right)^{-1} \hat{A}_{0}
$$

in $i\left[V(x+c(t)), A_{\lambda_{0}, \delta}\right]$ skillfully, in the proof of the Mourre estimate for $K$, where $\hat{A}_{0}$ is the generator of dilations on $X$. It is caused by that $\left|\left(\nabla_{X} V\right)(x+c(t))\right|$ does not vanish as $|x| \rightarrow \infty$, if $N \geq 3$. These are the issues in the future. Finally we note that if $V(x+c(t))$ is time-independent, one can obtain the Mourre estimate for $K$ by taking $\left(\lambda_{0}-\delta-D_{t}\right)^{-1} \hat{A}_{0}$ as a conjugate operator in the standard Mourre theory, even if $N \geq 3$. Hence we have a faint expectation that the factor $\left(\lambda_{0}-\delta-D_{t}\right)^{-1}$ will overcome the matter mentioned above.

Now we add a remark which may let this paper have a value. Very recently, the first author [3] has constructed a conjugate operator $A_{R}$ for $K$ in the standard Mourre theory when $N=3$, and obtained the Mourre estimate for $K . A_{R}$ is defined as

$$
\begin{aligned}
& A_{R}=\sum_{a \in \mathscr{A}} j_{a, R} A_{a} j_{a, R} ; \quad A_{a}=A_{\mathrm{AK}}^{a}+A_{\mathrm{Y}, a}, \\
& A_{\mathrm{AK}}^{a}=\left(3 \omega / 2-D_{t}\right)^{-1} \otimes \frac{\left\langle x^{a}, p^{a}\right\rangle+\left\langle p^{a}, x^{a}\right\rangle}{2}, \\
& A_{\mathrm{Y}, a}=\frac{\left\langle x_{a}, p_{a}\left(\omega / 4+\left(p_{a}\right)^{2} / 2\right)^{-1}\right\rangle+\left\langle\left(\omega / 4+\left(p_{a}\right)^{2} / 2\right)^{-1} p_{a}, x_{a}\right\rangle}{2},
\end{aligned}
$$


where $\mathscr{A}$ is the set of all cluster decompositions, and $\left\{j_{a, R}\right\}_{a \in \mathscr{A}}$ is a Graf type partition of unity of $X$ with sufficiently large $R$. Roughly speaking, our type conjugate operator $A_{\mathrm{AK}}^{a}$ is recognized as a conjugate operator for the Floquet Hamiltonian $K^{a}$ associated with the subsystem Hamiltonian $H^{a}(t)$, and the Yokoyama type conjugate operator $A_{\mathrm{Y}, a}$ can be recognized as a conjugate operator for the intercluster free Hamiltonian $\left(p_{a}\right)^{2} / 2$. Here we note that if $a=a_{\min }$, then $A_{\mathrm{Y}, a_{\min }}$ is recognized as a conjugate operator also for the free Floquet Hamiltonian $K_{a_{\min }}=$ $D_{t}+\left(p_{a_{\min }}\right)^{2} / 2$. Hence $A_{a}$ can be recognized as a conjugate operator for the Floquet Hamiltonian $K_{a}$ associated with the cluster Hamiltonian $H_{a}(t) . A_{R}$ can be constructed by gluing $A_{a}$ 's together with $\left\{j_{a, R}\right\}_{a \in \mathscr{A}}$. Therefore both [24] and this paper yield the first step of the above construction of a conjugate operator for $K$ with $N=3$.

\section{References}

[1] T. Adachi, Scattering theory for $N$-body quantum systems in a time-periodic electric field, Funkcial. Ekvac. 44 (2001), 335-376.

[2] T. Adachi, Asymptotic completeness for $N$-body quantum systems with long-range interactions in a time-periodic electric field, Comm. Math. Phys. 275 (2007), 443-477.

[3] T. Adachi, On the Mourre estimates for three-body Floquet Hamiltonians, preprint. https://arxiv.org/abs/1904.10190

[4] T. Adachi, T. Kimura and Y. Shimizu, Scattering theory for two-body quantum systems with singular potentials in a time-periodic electric field, J. Math. Phys. 51 (2010), 032103, 23 pp.

[5] W. O. Amrein, A. Boutet de Monvel and V. Georgescu, $C_{0}$-groups, commutator methods and spectral theory of $N$-body Hamiltonians, Progress in Mathematics 135, Birkhäuser Verlag, Basel, 1996.

[6] H. L. Cycon, R. G. Froese, W. Kirsch and B. Simon, Schrödinger operators with application to quantum mechanics and global geometry, Texts and Monographs in Physics, Springer-Verlag, Berlin, 1987.

[7] J. S. Howland, Stationary scattering theory for time-dependent Hamiltonians, Math. Ann. 207 (1974), 315-335.

[8] J. S. Howland, Scattering theory for Hamiltonians periodic in time, Indiana Univ. Math. J. 28 (1979), 471-494. 
[9] H. Kitada and K. Yajima, A scattering theory for time-dependent long-range potentials, Duke Math. J. 49 (1982), 341-376.

[10] E. L. Korotyaev, Scattering theory for a three-particle system with two-body interactions periodic in time, Theoret. and Math. Phys. 62 (1985), 163-170.

[11] Y. Kuwabara and K. Yajima, The limiting absorption principle for Schrödinger operators with long-range time-periodic potentials, J. Fac. Sci. Univ. Tokyo Sect. IA Math. 34 (1987), 833-851.

[12] E. Mourre, Absence of singular continuous spectrum for certain selfadjoint operators, Comm. Math. Phys. 78 (1981), 391-408.

[13] J. S. Møller, Two-body short-range systems in a time-periodic electric field, Duke Math. J. 105 (2000), 135-166.

[14] J. S. Møller and E. Skibsted, Spectral theory of time-periodic many-body systems, Adv. Math. 188 (2004), 137-221.

[15] S. Nakamura, Asymptotic completeness for three-body Schrödinger equations with time-periodic potentials, J. Fac. Sci. Univ. Tokyo Sect. IA Math. 33 (1986), 379-402.

[16] P. Perry, I. M. Sigal and B. Simon, Spectral analysis of $N$-body Schrödinger operators, Ann. of Math. (2) 114 (1981), 519-567.

[17] M. Reed and B. Simon, Methods of Modern Mathematical Physics I-IV, Academic Press.

[18] I. M. Sigal and A. Soffer, Long-range many-body scattering. Asymptotic clustering for Coulomb-type potentials, Invent. Math. 99 (1990), 115-143.

[19] B. Simon, Phase space analysis of simple scattering systems: extensions of some work of Enss, Duke Math. J. 46 (1979), 119-168.

[20] E. Skibsted, Spectral analysis of $N$-body systems coupled to a bosonic field, Rev. Math. Phys. 10 (1998), 989-1026.

[21] H. Tamura, Principle of limiting absorption for $N$-body Schrödinger operators - a remark on the commutator method, Lett. Math. Phys. 17 (1989), 31-36.

[22] K. Yajima, Scattering theory for Schrödinger equations with potentials periodic in time, J. Math. Soc. Japan 29 (1977), 729-743. 
[23] K. Yajima, Existence of solutions for Schrödinger evolution equations, Comm. Math. Phys. 110 (1987), 415-426.

[24] K. Yokoyama, Mourre theory for time-periodic systems, Nagoya Math. J. 149 (1998), 193-210. 